Enilma Marques Araujo' Simão Dias Vasconcelos ${ }^{2}$

\section{Biossegurança em Laboratórios Universitários: um Estudo de Caso na Universidade Federal de Pernambuco}

\author{
Biosafety in University Laboratories: \\ a Case Study at the Universidade \\ Federal de Pernambuco
}

'Mestre em Biologia Animal, Departamento de Zoologia da Universidade Federal de Pernambuco (UFPE)

${ }^{2}$ Professor Adjunto, Coordenador do Laboratório de Ensino de Zoologia do Departamento de Zoologia, Centro de Ciências Biológicas da Universidade Federal de Pernambuco (UFPE).
Embora seja uma das responsabilidades de um laboratório de ensino e pesquisa, a promoção da biossegurança como parte integrante da saúde ocupacional tem recebido limitada atenção em universidades brasileiras. Partindo desta necessidade, o presente trabalho analisou a adoção de procedimentos de proteção individual e coletiva nos sete laboratórios do Departamento de Micologia da UFPE através da aplicação de questionários junto aos responsáveis por cada um dos laboratórios, utilizando os critérios da CTNBio. Foram observadas algumas deficiências nos laboratórios, tais como ausência de extintores de incêndios, de protocolo de acidentes, chuveiros de emergência e limitada disponibilidade de avisos educativos para prevenção de acidentes. Por outro lado, detectou-se a preocupação com a correta armazenagem de produtos químicos e com a instalação elétrica/hidráulica dos laboratórios. Conclui-se que, de modo geral, a biossegurança é respeitada nos laboratórios analisados. Entretanto, é necessária uma maior conscientização dos usuários e uma inserção mais aprofundada do tema biossegurança nos cursos de Ciências Biológicas da UFPE.

Palavras-chave: biossegurança, fungos, laboratórios acadêmicos, riscos, saúde ocupacional.

Although biosafety is a major responsibility of research and teaching laboratories, its promotion as a component of occupational health has received limited attention in Brazilian universities. Due to this necessity, this study aimed to analyze the procedures for individual and collective protection at the seven laboratories of the Mycology Department at the UFPE, applying questionnaires based on CTNBio criteria to the laboratory coordinators. Some flaws were detected, such as the lack of fire extinguishers, accident protocol register, emergency showers, and scant warning and educational posters for accident prevention in the labs. On the other hand, the correct storage of chemicals and adequate electrical/ hydraulic facilities were observed. The overall biosafety procedures at the laboratories analyzed were adequate. However, a deeper user's awareness and a stronger integration of biosafety as a daily practice in Biology courses at the UFPE are needed.

Keywords: fungi, opportunistic infections, individual protection, risks, occupational health. 


\section{Introdução}

\section{Biossegurança: conceitos e imple- mentação de critérios}

A biossegurança compreende um conjunto de normas que visam prevenir, reduzir ou eliminar riscos inerentes às atividades de pesquisa, produção, ensino, desenvolvimento tecnológico e prestação de serviços, buscando eficiência sem comprometimento da saúde humana, de populações não-alvo e do equilíbrio do meio ambiente. Neste contexto, deve ser compreendida como parte fundamental da saúde ocupacional da educação profissionalizante e estimulada nas mais diversas formas de atividade humana.

No Brasil, a Comissão Técnica Nacional de Biossegurança (CTNBio), criada em 1995, estabelece a política nacional de biossegurança através de instruções normativas que devem ser cumpridas em todos os níveis. Entretanto, a ênfase da legislação - e conseqüente fiscalização - dos aspectos relacionados à biossegurança nos últimos anos parece ter se concentrado na utilização de organismos geneticamente modificados, especialmente os transgênicos. Com isso, questões fundamentais de saúde ocupacional, como a redução de riscos em locais de educação/formação de pessoal, recebem relativamente menor atenção. Esse aparente descuido assume proporções ainda mais graves quando ocorre em esferas responsáveis pela formação de recursos humanos especializados, tais como instituições de ensino superior.

O cumprimento dos parâmetros de biossegurança é particularmente crítico em laboratórios de pesquisa e ensino devido à alta rotatividade de usuários - professores, pesquisadores, estagiários, alunos de graduação e pós-graduação e funcionários de manutenção (Hirata, 2002). As diversas atividades didáticas e experimentais expõem os usuários a variados riscos associados à manipulação de instrumentos perfuro-cortantes, produtos químicos (solventes, tóxicos, abrasivos, irritantes, inflamáveis, voláteis, cáusticos, entre outros), à exposição a incêndios, ruídos, eletricidade, radiação e, especialmente, a microorganismos patogênicos ao homem (Hirata, 2002).

\section{Riscos associados aos laboratórios de ensino e pesquisa sobre micror- ganismos}

As características peculiares dos microorganismos, como seu grau de patogenicidade, poder de invasão, resistência a processos de esterilização, virulência e capacidade mutagênica, exigem cuidados especiais em sua manipulação (Teixeira \& Valle, 1996). Num laboratório de pesquisa de fungos, por exemplo, a exposição pode ocorrer principalmente por inalação de partículas fúngicas, por ingestão acidental ou pela inoculação direta como resultado de acidentes com vidraria ou agulhas (Collins, 1983). Usuários de um laboratório de micologia estão submetidos a um elevado risco de adquirir infecções micóticas, as quais correspondem a cerca de $10 \%$ das infecções originadas em laboratórios de pesquisa (Fiocruz, 1998).

As micoses podem ser classificadas em superficiais, cutâneas, subcutâneas, sistêmicas, e oportunistas (Kobaiashi \& Medoff, 2002). Micoses superficiais limitam-se às camadas externas e "mortas" da pele e na maioria das vezes não sensibilizam imunologicamente 0 indivíduo e, quando o fazem, provocam infecções leves. Entre os agentes etiológicos destacam-se as espécies pertencentes aos gêneros Exophiala, Malassezia e Trichosporon. Micoses cutâneas, embora restritas à epiderme, podem desencadear resposta imunocelular, sensibilizando o hospedeiro. Os principais representantes são espécies dos gêneros Epidermophyton, Microsporum e Tricophyton. Micoses subcutâneas, provocadas por fungos saprófitas associados a uma inoculação traumática, atingem músculos, ossos e tecido conjuntivo. É o caso dos gêneros Cladosporium, Fonsecae e Sporothrix. Micoses sistêmicas podem atingir qualquer tecido ou órgão interno e são provocadas por fungos inalados, infectando inicialmente o pulmão. Fungos dos gêneros Blastomyces, Coccidioides e Histoplasma estão neste grupo capaz de infectar indivíduos com o sistema imunológico competente. Já nas micoses oportunistas, o fungo possui baixa patogenicidade em condições normais e não induz a doenças, a não ser que o hospedeiro esteja imunodeprimido. Este grupo inclui espécies dos gêneros Aspergillus, Candida e Mucro (Kobaiashi \& Medoff, 2002).

A CTNBio classifica os patógenos em quatro classes de risco e níveis crescentes de biossegurança (NB1, NB2, NB3 e NB4) que variam quanto ao grau de contenção do organismo e à complexidade do nível de proteção (CTNBio, 1997). A Classe de Risco 1 abrange os fungos fitopatogênicos e os fun- 
gos que não causam doença em seres humanos ou outros animais. A Classe 2 possui risco moderado para o homem e outros animais e risco limitado para a comunidade. Inclui espécies dos gêneros Aspergillus, Blastomyces, Candida, Cladosporium, Cryptococcus, Dactylaria, Epidermophyton, Exophiala, Fonsecaea, Microsporum, Paracoccidoides, Penicillium e Trichophyton. Já a Classe 3 representa elevado risco para o homem e os animais, embora ofereça risco limitado à comunidade por existirem medidas de tratamento e prevenção. Os fungos deste grupo são Coccidioides immittis e Histoplasma capsulatum. Na Classe de Risco 4 estão os patógenos letais ao homem, facilmente transmissíveis e sem medidas preventivas ou de tratamento conhecidas até o momento. Não há fungos classificados pela CTNBio neste grupo.

\section{Inserção da biossegurança no con- texto educacional: o caso da Univer- sidade Federal de Pernambuco}

Apesar de sua evidente importância, questões relacionadas à biossegurança ainda são ignoradas em diversos cursos das áreas de Saúde e Biologia em todo o país. Por exemplo, o Centro de Ciências Biológicas (CCB) da Universidade Federal de Pernambuco, através de seus dez departamentos, oferece quatro cursos de graduação, nove cursos de mestrado e três de doutorado. Uma breve análise do quadro de disciplinas obrigatórias e eletivas oferecidas nos cursos de pós-graduação revela a inexistência de disciplinas voltadas especificamente para a discussão de temas relacionados à biossegurança.

Dos quatro cursos de graduação oferecidos no CCB - os bacharelados em Ciências Biológicas, Ciências Biomédicas e Ciências Ambientais e a licenciatura em Ciências Biológicas -, apenas o curso de bacharelado em Ciências Biomédicas oferece regularmente a disciplina - eletiva - sobre biossegurança em sua grade curricular. No bacharelado em Ciências Biológicas, tal assunto é pouco explorado em uma disciplina eletiva raramente oferecida. O curso de Ciências Ambientais modalidade do bacharelado em Ciências $\mathrm{Bi}$ ológicas - é um curso em fase de implementação, iniciado em 2002. Surpreendentemente, apesar da preocupação com a transdisciplinaridade e a atualização do perfil do graduando, tópicos de biossegurança não foram diretamente contemplados na grade curricular. Na licenciatura em Ciências Biológicas não há qualquer disciplina com este conteúdo na estrutura curricular, sendo este tema largamente ignorado.

A relevância da adoção de procedimentos adequados de biossegurança em laboratórios de ensino e pesquisa associada à necessidade da inserção do tema de forma aplicada nos cursos de Ciências Biológicas e Ambientais estimula o debate e a pesquisa sobre formas de implementação desta prática no cotidiano acadêmico. Partindo dessa necessidade, o presente trabalho teve duplo objetivo: em primeiro lugar, buscou-se analisar os procedimentos de biossegurança adotados nos laboratórios de ensino/pesquisa do Centro de Ciências Biológicas da UFPE a fim de se detectar a que tipos de risco os profissionais em formação estão submetidos. Ao longo da pesquisa, foi dada ênfase na questão do risco biológico, na proteção individual do usuário e nos procedimentos de manipulação e descarte de microorganismos. Em segundo lugar, visou envolver os estudantes de graduação no processo de investigação do cumprimento das normas de biossegurança em seu cotidiano para que os mesmos assumam uma postura responsável e crítica. Com esta prática buscou-se promover o acesso dos graduandos a informações contextualizadas e estimular o debate sobre a necessidade da promoção da saúde ocupacional no ambiente acadêmico.

O objetivo não foi meramente detectar faIhas, mas, principalmente, iniciar um processo de percepção constante de riscos associados à prática de laboratório. Como objeto de estudo inicial, os estudantes investigaram um dos dez departamentos do CCB da UFPE, o Departamento de Micologia, onde são pesquisados fungos de importância econômica, agrícola e médica.

\section{Metodologia}

A pesquisa foi realizada como atividade integrante da disciplina eletiva "Redação e Apresentação Científica", do bacharelado em Ciências Biológicas, a qual estimula a prática de projetos de pesquisa em grupo. Um grupo de alunos optou pelo tema da biossegurança em laboratórios de ensino e pesquisa do CCB da UFPE, com ênfase na saúde ocupacional de seus usuários. Buscou-se verificar o cumprimento das normas de biossegurança e detectar se a universidade estava cumprindo seu papel (in)formador. 
Como modelo, escolhido em parte pelo fato de infecções causadas por fungos serem um dos problemas mais freqüentes em laboratórios microbiológicos, pela facilidade de acesso e pelo grande número de usuários, escoIheu-se o Departamento de Micologia. Esse departamento conta com as seguintes divisões: Laboratório de Biologia Molecular, Coleção Didático-Científica (Micoteca), Laboratório de Controle Biológico, Laboratório de Micorrizas, Laboratório de Fitopatologia, Laboratório de Imunodiagnóstico e Laboratório de Micologia Médica. Estes dois últimos utilizam as mesmas instalações e têm funções complementares, razão pela qual foram agrupados em um Laboratório de Micologia Clínica. Todos os laboratórios são utilizados diariamente por professores-pesquisadores, técnicos e alunos, em diferentes graus de acesso.

Baseando-se em uma visita prévia para reconhecimento da realidade de cada laboratório, foi elaborado um questionário a partir das sugestões de segurança laboratorial do Manual de Biossegurança de Hirata \& Mancini Filho (2002). Nos meses de janeiro e fevereiro de 2003, os questionários foram respondidos pelos professores-pesquisadores responsáveis por cada laboratório. Embora o enfoque tenha sido a contaminação com microorganismos, também foram abordados tópicos como uso e armazenamento de ma- teriais de risco, equipamentos de proteção individual e coletiva, presença de kits de primeiros socorros, sinalização das situações de risco, entre outros. As respostas foram analisadas de acordo com os critérios propostos pela Comissão Técnica Nacional de Biossegurança (1997), por manuais de laboratório de instituições de referência nacional, como o da Fiocruz (1998), e por bibliografia especializada (Hirata \& Mancini Filho, 2002). A todos os entrevistados foi comunicada a intenção de divulgar publicamente o resultado do trabalho e solicitada a permissão para citar os nomes dos laboratórios.

\section{Resultados e discussão}

\section{A promoção da biossegurança nos laboratórios de ensino e pesquisa}

A partir das respostas dos questionários, foi possivel traçar um perfil das situações de risco presentes em cada laboratório. De modo geral, o risco biológico dos fungos pesquisados no Departamento de Micologia é baixo. Com exceção do Laboratório de Micologia Clínica e da Coleção Didático-Científica (Micoteca), os fungos estudados encontramse nas classes de menor risco. Isso porque nos demais laboratórios são pesquisados, por exemplo, fitopatógenos e entomopatógenos que, de modo geral, não são patogênicos ao homem (Tabela 1).

Tabela 1 Perfil dos laboratórios analisados no Departamento de Micologia da UFPE em janeiro-fevereiro de 2003 e grau de periculosidade dos fungos pesquisados.

\begin{tabular}{l|l|c}
\hline \multicolumn{1}{c|}{ Laboratório } & \multicolumn{1}{|c}{$\begin{array}{c}\text { Principais tipos de fungos } \\
\text { estudados }\end{array}$} & $\begin{array}{c}\text { Grau de risco do } \\
\text { fungo mais patogênico } \\
\text { ao homem }\end{array}$ \\
\hline Biologia Molecular & $\begin{array}{l}\text { Fitopatógenos em geral e patógenos } \\
\text { humanos de grau leve }\end{array}$ & 2 \\
\hline Micoteca & Todos os tipos de fungos & 3 \\
\hline Controle Biológico & Fungos entomopatógenos & 1 \\
\hline Micorrizas & Micorrizas & 2 \\
\hline Fungos Fitopatogênicos & Fitopatógenos e suas toxinas & 3 \\
\hline Micologia Clínica & Fungos de importância médica & 1 \\
\hline
\end{tabular}


As repostas dos questionários estão sintetizadas na Tabela 2. Pode-se afirmar que não há grandes diferenças na adoção de procedimentos de biossegurança entre os laboratórios, havendo, no entanto, algumas deficiências - em graus variados - em cada um deles. Os cuidados com a armazenagem de substâncias químicas corrosivas, voláteis e inflamáveis e com a instalação hidráulica/elétrica são adotados pela maioria dos laboratórios. Evidentemente, a correta armazenagem de líquidos reduz o risco de acidentes, desde quebra de vasilhames à mistura de material inflamável com não inflamável. A possível ocorrência de incêndios seria bastante agravada, já que nenhum dos laboratórios possui extintores próprios, havendo apenas extintores na área comum e, mesmo assim, em quantidade e condições inadequadas para atender às exigências mínimas de segurança em suas instalações. Observouse ainda a ausência de chuveiros de emergência e lava-olhos na maioria dos laboratórios.

Tabela 2 Adoção de práticas de biossegurança nos laboratórios do Departamento de Micologia da UFPE, de acordo com entrevistas realizadas em janeiro-fevereiro de 2003.

\begin{tabular}{|c|c|c|c|c|c|c|}
\hline ITEM & $\begin{array}{c}\text { LAB. BIOLOGIA } \\
\text { MOLECULAR }\end{array}$ & MICOTECA & $\begin{array}{l}\text { CONTOLE } \\
\text { BIOLÓGICO }\end{array}$ & MICORRIZA & $\begin{array}{c}\text { FITO- } \\
\text { PATOLOGIA }\end{array}$ & $\begin{array}{l}\text { MICOLOGIA } \\
\text { CLÍNICA }\end{array}$ \\
\hline $\begin{array}{l}\text { ARMAZENAGEM SEGURA DE } \\
\text { MATERIAL DE RISCO }\end{array}$ & & & & & & \\
\hline $\begin{array}{l}\text { DISPOSIÇĀO SEGURA DOS } \\
\text { MATERIAIS E EQUIPAMENTOS }\end{array}$ & & & & & & \\
\hline $\begin{array}{l}\text { RECURSOS ELÉTRICOS E HIIDR } \\
\text { EM CONDIÇŌES SATISFATÓRIA }\end{array}$ & & & & & & \\
\hline $\begin{array}{l}\text { ARMAZENAGEM CORRETA DE } \\
\text { LíQUIDO INFLAMÁVEL }\end{array}$ & & & & & & \\
\hline $\begin{array}{l}\text { PRESENÇA DE EXTINTORES } \\
\text { DE INCENDDIOS }\end{array}$ & & & & & & \\
\hline $\begin{array}{l}\text { PROTEÇÃO INDIVIDUAL } \\
\text { NO TRABALHO }\end{array}$ & & & & & & \\
\hline $\begin{array}{l}\text { PRESENÇA DE KITS DE } \\
\text { PRIMEIROS SOCORROS }\end{array}$ & & & & & & \\
\hline $\begin{array}{l}\text { LISTA DE EMERGËNCIA } \\
\text { EM ACESSO FÁCIL }\end{array}$ & & & & & & \\
\hline $\begin{array}{l}\text { INFORMAÇŌES A MULHERES } \\
\text { EM IDADE FÉRTIL }\end{array}$ & & & & & & \\
\hline $\begin{array}{l}\text { PROTOCOLO PARA REGISTRO } \\
\text { DE ACIDENTES }\end{array}$ & & & & & & \\
\hline $\begin{array}{l}\text { AVISOS PARA PREVENÇÃO DE } \\
\text { ACIDENTES NO LABORATÓRIO }\end{array}$ & & & & & & \\
\hline
\end{tabular}

$$
\left\{\begin{array}{l}
\text { Sim } \\
\text { Não }
\end{array}\right.
$$

O uso constante de equipamento de proteção individual, incluindo aventais, máscaras e luvas, foi registrado em $50 \%$ dos laboratórios (Tabela 2). Nos demais, segundo os entrevistados, roupas e aparatos apropriados são utilizados apenas durante o manuseio de material biológico ou considerado de risco (reagentes voláteis e corrosivos, por exemplo). Observou-se que uso de máscaras de proteção facial é um procedimento pouco freqüente no departamento.

Em nenhum dos laboratórios visitados havia estojos de primeiros socorros ou protocolo para registro de doenças e/ou acidentes ocorridos. Adicionalmente, com exceção do Laboratório de Micorrizas, não são disponibilizadas, em locais de fácil acesso, listas com telefones/contatos para atendimento em caso de emergência. Como agravante, apenas os laboratórios de Biologia Molecular e Micologia Clínica disponibilizam cartazes educativos para prevenção de acidentes de trabalho (Tabela 2). Por outro lado, a maioria dos laboratórios mantém informações sobre procedimentos e substâncias de risco para mulheres em idade fértil.

A ausência de protocolo para registro de acidentes constitui uma grave falha em um laboratório de ensino e pesquisa. Entretanto, - fato de não ter sido registrado, segundo os responsáveis, nenhum tipo de acidente (contaminação, queimadura, corte, processo alérgico ou outro) entre os usuários dos laboratórios não deve subestimar o risco das atividades desenvolvidas. Infere-se que o treinamento dos novos alunos, como estagiários 
de pesquisa e alunos de pós-graduação, é predominantemente informal e baseado no exemplo dado pelos pesquisadores mais experientes. Aparentemente, tal treinamento tem sido suficiente até o momento para evitar acidentes com os usuários.

Apesar das imperfeições detectadas, de modo geral, o Departamento de Micologia obedece aos padrões de biossegurança em seus laboratórios, acatando a maioria das recomendações da CTNBio. No entanto, devese dar maior ênfase à adoção de equipamentos de proteção individual e coletiva para garantir proteção adequada, inclusive contra fungos considerados de baixo risco. A ausência de condições apropriadas para combate a incêndios - incluindo a localização, 0 número e a validade dos extintores e o treinamento periódico de pessoal para lidar com situações de emergência - precisa ser urgentemente solucionada. Parcerias com os órgãos competentes (por exemplo, o Corpo de Bombeiros local) seriam alternativas de baixo custo e enorme repercussão.

As situações de risco em um laboratório de ensino e pesquisa de fungos não se limitam, naturalmente, aos microorganismos-alvo. Segundo o CDC (1993), diversas espécies de fungos presentes no ambiente têm causado sérias infecções em hospedeiros imunocompetentes que inalaram ou se inocularam acidentalmente por via subcutânea (Fiocruz, 1998). Destacam-se espécies dos gêneros Cladosporium, Penicillium e Exophiala. Além desses, há fungos considerados emergentes, como patógenos oportunistas, os quais incluem espécies dos gêneros Beauveria, Coprinus, Fusarium e Rhizoctonia (Torres-Rodriguez, 1996; Fiocruz, 1998). Embora o foco deste trabalho tenha sido o risco associado ao contato com microorganismos, há, naturalmente, inúmeros outros elementos de natureza não-biológica que oferecem risco à saúde do usuário. Estes incluem desde uso, armazenamento e descarte inadequados de substâncias químicas (corantes, ácidos, bases, solventes etc.) à manipulação de objetos perfuro-cortantes, passando pela probabilidade de choques elétricos e exposição a excessivas temperatura, umidade e radiações - ionizantes ou não. O risco é inversamente proporcional ao nível de informação e capacitação do usuário.

\section{Inserção da biossegurança como prática dos projetos pedagógicos}

As imperfeições detectadas nos laboratórios decorrem primordialmente de dois obstácu- los: em primeiro lugar, registra-se a lastimável situação das universidades públicas no que se refere aos recursos para manutenção dos laboratórios. Com os orçamentos praticamente congelados há vários anos e a escassez de projetos de financiamento específicos para capacitação de recursos humanos em biossegurança, os professores responsáveis pelos laboratórios precisam buscar recursos de outras fontes para aquisição de material e manutenção de equipamentos que garantam padrões mínimos de segurança. Como nem sempre isso é possível, a parcimônia na aquisição de material e equipamento de proteção, nos gastos com a manutenção de instalações e equipamentos e ainda com atividades de capacitação acaba por aumentar o risco à saúde dos usuários.

O outro problema resulta do fato de a biossegurança como prática diária em situações de pesquisa e ensino ser ainda incipiente no Brasil. A inserção da biossegurança na grade curricular dos cursos de Ciências Biológicas e afins parece ser pouco compreendida até mesmo em universidades e órgãos de fomento. Os alunos de graduação, por serem mais inexperientes e, na maioria das vezes, desempenharem atividades de curta duração, estão particularmente vulneráveis a acidentes. O aluno deve, pois, ser preparado para exercer seu senso crítico e investigativo a fim de detectar situações de risco em seu cotidiano acadêmico. Mas será que a grade curricular, as metodologias de ensino e até a orientação disponibilizada nos estágios de pesquisa promovem esse tipo de olhar?

Como destacam Consiglieri e Hirata (2002), é necessário que os alunos sejam previamente conscientizados sobre a quais riscos serão submetidos no laboratório e treinados sobre como proceder em casos de acidentes. Os aspectos gerais devem contemplar a orientação quanto à indumentária (uso de avental, sapatos baixos e fechados, calças compridas), ao emprego correto dos equipamentos de proteção individual (máscaras, luvas, toucas, óculos), aos procedimentos de manipulação de substâncias e ao comportamento no laboratório (Consiglieri \& Hirata, 2002).

\section{Considerações finais}

A promoção da segurança em ambientes acadêmicos é de responsabilidade pessoal, da chefia do laboratório e da instituição. Assim, cada laboratório deve estabelecer medidas preventivas adequadas aos respec- 
tivos agentes pesquisados, informando aos usuários o potencial de risco e as respectivas medidas de proteção necessárias (McGinnis, 1980). Aos docentes cabe ainda avaliar o risco em função do número de alunos e da qualidade das instalações, pois laboratórios superlotados e instalações deficientes tendem a potencializar o risco de acidentes (Consiglieri \& Hirata, 2002). Laboratórios de ensino e pesquisa das universidades deveriam, teoricamente, funcionar como modelos para o ensino-aprendizagem de normas de proteção individual, coletiva e ambiental. Por serem centros formadores nas mais diversas profissões, a promoção da saúde ocupacional deveria ser parte integrante do cotidiano das situações de ensino, especialmente nas aulas práticas.

Uma rápida visita aos laboratórios universitários brasileiros revela que a maioria não respeita integralmente as normas de biossegurança. Felizmente, observa-se que tal situação tem melhorado significativamente nos últimos anos, estimulada por organizações como a Associação Nacional de Biossegurança (ANBio). No âmbito da Universidade Federal de Pernambuco, destaca-se o curso anual de capacitação de agentes de biossegurança promovido pelo Departamento de Antibióticos do Centro de Ciências Biológicas, que, com apoio do Ministério da Ciência e Tecnologia/CNPq, vem formando agentes multiplicadores entre estudantes, professores, pesquisadores e representantes de organizações públicas, particulares e nãogovernamentais. Com a expansão das linhas de pesquisa biológica, incluindo a provável utilização em maior escala de organismos geneticamente modificados e a busca por vacinas contra agentes de alta virulência, é provável que novas situações de risco sejam rapidamente criadas em laboratório. O acompanhamento dessas situações, de maneira integrada envolvendo biólogos, advogados, médicos, engenheiros, representantes do governo e da sociedade civil, entre outros, é essencial para garantir a saúde coletiva dos cidadãos expostos a situações de perigo.

Com o presente trabalho não se pretendeu "fiscalizar" laboratórios, mas sim desenvolver com os estudantes a prática de projetos de pesquisa que insiram a biossegurança como componente da formação acadêmica. A observação da realidade concreta dos laboratórios através da prática de projetos gerov entre os alunos a necessidade de adquirir conhecimento para criar instrumentos de diagnóstico e ação, promovendo uma postura mais crítica em relação as suas próprias atitudes como futuros pesquisadores. Para que a questão da biossegurança seja abordada de forma integrada, é fundamental que seus princípios sejam incorporados à prática de laboratório, desde o início da vida acadêmica do estudante.

\section{Agradecimentos}

Os autores agradecem às doutoras Cristina Souza Motta, Elza Luna Lima, Leonor Costa Maia, Neiva Tinti Oliveira, Oliane Magalhães e Severina Torres Barros pelo conhecimento socializado durante a pesquisa e aos professores Sidney Bastos, Oliane Magalhães e Glícia Calazans, todos da Universidade $\mathrm{Fe}$ deral de Pernambuco, pela leitura crítica do manuscrito. Sinceros agradecimentos à Tatiana Cristina Azevedo Freitas e Ng Haig They pela valiosa participação na coleta de dados.

\section{Referências Bibliográficas}

\author{
COLLINS, C. H. Laboratory-acquired \\ infections. London: Butteworth \& Co. \\ Ltd., 1983, $456 \mathrm{p}$. \\ CONSIGLIERI, V. O.; \& HANATA, R. D. C. \\ Biossegurança em laboratórios de ensino \\ e da área de Saúde. In. HIRATA, M. H.; \& \\ MANCINI FILHO, J. Manual de \\ Biossegurança, São Paulo: Manole, \\ 2002, pp. 47-55.
}

CTNBio - Comissão Técnica Nacional de Biossegurança. Instrução Normativa № 7, publicada no Diário Oficial da União de 09/06/1997, pp. 11827-11833.

FIOCRUZ. Procedimentos para a manipulação de microorganismos patogênicos e/ou recombinantes na FIOCRUZ. Rio de Janeiro: Editora da Fiocruz, 1998, $166 \mathrm{p}$. 
HIRATA, M. H.; O laboratório de ensino e pesquisa e seus riscos. In: HIRATA, $M$. $H_{\text {.; }}$ \& MANCINI FILHO, J. Manual de Biossegurança. São Paulo: Manole, 2002, pp. 1-19.

HIRATA, M. H.; \& MANCINI FILHO, J. Manual de Biossegurança. São Paulo: Manole, 2002, 423 p.

KOBAIASHI, G. S.; \& MEDOFF, G. Introdução aos fungos e as micoses. In: SHAECHTER, M; ENGLEBERG, N. C.; \& EISENGLEIN, B. I. (eds.) Microbiologia: mecanismos de doenças infecciosas. 3 ed. São Paulo: Guanabara Koogan, 2002, pp. 373-379
McGINNIS, M. R. Laboratory Handbook of Medical Mycology. New York: Academic Press, 1980, 498 p.

TEIXEIRA, P.; \& VALLE, S. Biossegurança: uma abordagem multidisciplinar. Rio de Janeiro: Editora da Fiocruz, 1996, $362 \mathrm{p}$

TEIXEIRA, P.; \& VALLE, S. Riscos Biológicos em Laboratório. In: VALLE, S.; \& TELLES, J. L. (orgs.) Bioética e Biorrisco. Rio de Janeiro: Interciência, 2003, pp. 205-215.

TORRES-RODRIGUEZ, J. M. Nuevos hongos patógenos oportunistas emergentes. $\mathbf{R e}$ vista Iberoamericana de Micologia, 12: 30-45, 1996. 